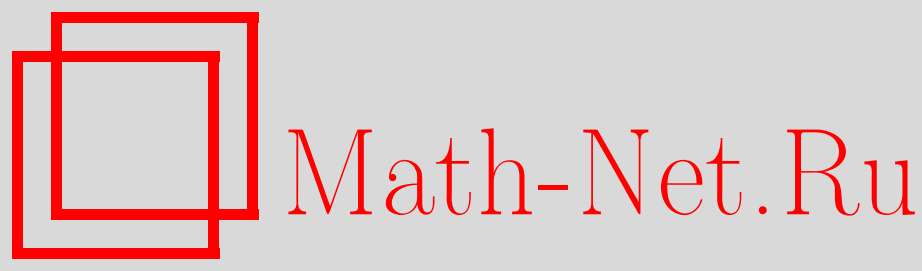

У. Лаха, С. Рэй, С. Панда, Я. Бхои, Преобразования Лапласа хюльтеновской функции Грина и их применение к задаче потенциального рассеяния, ТМФ, 2017, том 193, номер 1, 104-114

DOI: https://doi.org/10.4213/tmf9289

Использование Общероссийского математического портала Math-Net.Ru подразумевает, что вы прочитали и согласны с пользовательским соглашением http://www . mathnet.ru/rus/agreement

Параметры загрузки:

IP : 3.93.64.190

26 апреля 2023 г., $11: 30: 51$

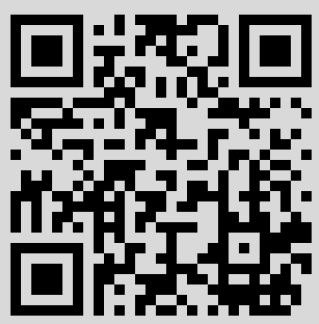




\title{
ПРЕОБРАЗОВАНИЯ ЛАПЛАСА ХЮЛЬТЕНОВСКОЙ ФУНКЦИИ ГРИНА И ИХ ПРИМЕНЕНИЕ К ЗАДАЧЕ ПОТЕНЦИАЛЬНОГО РАССЕЯНИЯ
}

\begin{abstract}
Выведены и записаны в максимально редуцированном виде замкнутые представления одно- и двукратного преобразований Лапласа хюльтеновской функции Грина уходящей волны, умноженной на потенциал Ямагучи. Выражения для двукратного преобразования используются при вычислении низкоэнергетического фазового сдвига при упругом рассеянии в системах $\alpha-$ нуклон, $\alpha-\mathrm{He}^{3}$ и $\alpha-\mathrm{H}^{3}$. Результаты расчета хорошо согласуются с экспериментальными данными.
\end{abstract}

Ключевые слова: хюльтеновская функция Грина, интегральные преобразования хюльтеновской функции Грина, расчет сдвига фазы рассеяния, системы $\alpha$-нуклон и $\alpha$-ядро.

DOI: https://doi.org/10.4213/tmf9289

\section{1. ВВЕДЕНИЕ}

В атомной физике и физике плазмы экранированный и усеченный кулоновские потенциалы часто используются для вычисления наблюдаемых рассеяния и основного состояния. Основное преимущество хюльтеновского потенциала заключается в том, что он позволяет получить аналитические решения для S-волн. C применением целых аналитических регулярных и нерегулярных решений уравнения Шредингера с хюльтеновским потенциалом в случае $\ell=0$ были построены интегральные преобразования хюльтеновской функции Грина уходящей волны, умноженной на некоторый специальный близкодействующий потенциал. Выражения для двукратных интегральных преобразований хюльтеновской функции Грина уходящей волны используются при вычислении сдвига фазы рассеяния в системах $\alpha-$ нуклон и $\alpha$-ядро. Показано, что в пределе отсутствия экранирования, когда хюльтеновский потенциал переходит в кулоновский, модифицированные хюльтеновские интегральные преобразования совпадают со своим кулоновским аналогами.

* Department of Physics, National Institute of Technology, Jamshedpur, Jharkhand, India. E-mail: ujjwal.laha@gmail.com

${ }^{\dagger}$ Department of Physics, Government College of Engineering, Kalahandi, Odisha, India. E-mail: jskbhoi@gmail.com 
При энергии в системе центра масс $E=k^{2}+i \varepsilon$ функция Грина уходящей волны для движения в потенциале, являющемся суммой потенциалов Хюльтена [1] и Ямагучи [2], удовлетворяет интегро-дифференциальному уравнению

$$
\left[\frac{d^{2}}{d r^{2}}+k^{2}-V_{0} \frac{e^{-r / a}}{1-e^{-r / a}}\right] G_{\mathrm{HY}}^{(+)}\left(r, r^{\prime}\right)-\lambda g(r) \int_{0}^{\infty} d r^{\prime} g\left(r^{\prime}\right) G_{\mathrm{HY}}^{(+)}\left(r, r^{\prime}\right)=\delta\left(r-r^{\prime}\right),
$$

где $\lambda$ - константа связи и $g(r)$ - потенциал Ямагучи, а $V_{0}$ и $a$ - сила и радиус экранирования атомного потенциала Хюльтена. Интегральное уравнение, отвечающее уравнению (1), записывается как

$$
G_{\mathrm{HY}}^{(+)}\left(r, r^{\prime}\right)=G_{\mathrm{H}}^{(+)}\left(r, r^{\prime}\right)+\lambda d(r, k) \int_{0}^{\infty} d r g(r) G_{\mathrm{H}}^{(+)}\left(r, r^{\prime}\right),
$$

где

$$
d(r, k)=\int_{0}^{\infty} d r^{\prime} g\left(r^{\prime}\right) G_{\mathrm{HY}}^{(+)}\left(r, r^{\prime}\right) .
$$

Уравнение (2) легко решается, получаем

$$
G_{\mathrm{HY}}^{(+)}\left(r, r^{\prime}\right)=G_{\mathrm{H}}^{(+)}\left(r, r^{\prime}\right)+\lambda \frac{G_{\mathrm{H}}^{(+)}\left(\alpha, r^{\prime}\right) G_{\mathrm{H}}^{(+)}(\beta, r)}{1-\lambda G_{\mathrm{H}}^{(+)}(\alpha, \beta)},
$$

где

$$
\begin{aligned}
G_{\mathrm{H}}^{(+)}\left(\alpha, r^{\prime}\right) & =\int_{0}^{\infty} d r g(r) G_{\mathrm{H}}^{(+)}\left(r, r^{\prime}\right), \\
G_{\mathrm{H}}^{(+)}(\beta, r) & =\int_{0}^{\infty} d r^{\prime} g\left(r^{\prime}\right) G_{\mathrm{H}}^{(+)}\left(r, r^{\prime}\right), \\
G_{\mathrm{H}}^{(+)}(\alpha, \beta) & =\int_{0}^{\infty} d r g(r) \int_{0}^{\infty} d r^{\prime} g\left(r^{\prime}\right) G_{\mathrm{H}}^{(+)}\left(r, r^{\prime}\right) .
\end{aligned}
$$

Цель настоящей статьи - получение точных аналитических выражений для однои двукратных преобразований хюльтеновской функции Грина $G_{\mathrm{H}}^{(+)}\left(r, r^{\prime}\right)$ с потенциалом Ямагучи. В разделе 2 мы получаем в замкнутом виде формулы для преобразования Лапласа хюльтеновской функции Грина. Раздел 3 посвящен исследованию кулоновского предела наших результатов и проверке значимости полученных выражений на некоторых модельных расчетах. В разделе 4 мы подводим итоги.

\section{2. ВЫРАЖЕНИЯ ДЛЯ ФУНКЦИЙ $G_{\mathrm{H}}^{(+)}(r, \beta)$ И $G_{\mathrm{H}}^{(+)}(\alpha, \beta)$}

Потенциал Хюльтена позволяет получить аналитические решения уравнения Шредингера только для S-волн. Поэтому мы будем работать только с S-волновой функцией Грина. При энергии в системе центра масс $E=k^{2}+i \varepsilon$ хюльтеновская функция Грина уходящей волны удовлетворяет неоднородному дифференциальному уравнению [3]

$$
\left(\frac{d^{2}}{d r^{2}}+k^{2}-V_{0} \frac{e^{-r / a}}{1-e^{-r / a}}\right) G_{\mathrm{H}}^{(+)}\left(r, r^{\prime}\right)=\delta\left(r-r^{\prime}\right),
$$

где параметр $V_{0}$ потенциала Хюльтена действителен и положителен. Используя в (7) преобразование

$$
G_{\mathrm{H}}^{(+)}\left(r, r^{\prime}\right)=a e^{i k r}\left(1-e^{-r / a}\right) F\left(r, r^{\prime}\right),
$$


мы имеем

$$
\begin{aligned}
& \left(1-e^{-r / a}\right) \frac{d^{2} F}{d r^{2}}+\frac{1}{a}\left[2 i k a+(2-2 i k a) e^{-r / a}\right] \frac{d F}{d r}+\frac{1}{a^{2}}\left(2 i k a-1-V_{0} a^{2}\right) e^{-r / a} F= \\
& =\frac{1}{a} e^{-i k r} \delta\left(r-r^{\prime}\right) .
\end{aligned}
$$

Совершая в (9) преобразование Лапласа $\left(r^{\prime} \rightarrow \beta\right)$ и подстановку $x=1-e^{-r / a}$, мы получаем

$$
x(1-x) \frac{d^{2} \bar{F}}{d x^{2}}+[2-(3-2 i a k) x] \frac{d \bar{F}}{d x}+\left(2 i a k-1-V_{0} a^{2}\right) \bar{F}=a(1-x)^{(\beta+i k) a-1},
$$

где $\bar{F}(r, \beta)=L\left\{F\left(r, r^{\prime}\right) ; r^{\prime} \rightarrow \beta\right\}$. Сравнивая уравнение (10) с неоднородным гипергеометрическим уравнением Гаусса [4], [5]

$$
z(1-z) \frac{d^{2} y}{d z^{2}}+\left[c_{1}-\left(a_{1}+b_{1}+1\right) z\right] \frac{d y}{d z}-a_{1} b_{1} y=z^{\sigma-1}(1-\rho z)^{\tau-1}
$$

в котором $a_{1}, b_{1}, c_{1}, \rho, \tau$ и $\sigma$ - постоянные, получаем полный интеграл уравнения (10):

$$
\begin{aligned}
\bar{F}(r, \beta)= & R_{12} F_{1}\left(1+A, 1+B ; 2 ; 1-e^{-r / a}\right)+ \\
& +\left.R_{2}\left(1-e^{-r / a}\right)^{-1}{ }_{2} F_{1 \varepsilon}\left(A, B ; \varepsilon ; 1-e^{-r / a}\right)\right|_{\varepsilon \rightarrow 0}+ \\
& +a \sum_{n=0}^{\infty} \frac{\Gamma(n+1-(\beta+i k) a)}{\Gamma(1-(\beta+i k) a) n !} f_{n+1}\left(A+1, B+1 ; 2 ; 1-e^{-r / a}\right),
\end{aligned}
$$

где [4]

$$
f_{n}(a, b ; c ; z)=\frac{z^{n}}{n(n+c-1)}{ }_{3} F_{2}(1, n+a, n+b ; n+1, n+c ; z) .
$$

Ряд в (12) сходится при $|z|<1$; при $|z|=1$ он сходится, если $\operatorname{Re}(c-a-b)>0$. В уравнении (12) $R_{1}$ и $R_{2}$ - две произвольные постоянные, которые находятся из граничных условий, и

$$
A=-i a k+i a\left(k^{2}+V_{0}\right)^{1 / 2}, \quad B=-i a k-i a\left(k^{2}+V_{0}\right)^{1 / 2} .
$$

Заметим также, что независимые решения однородного уравнения, отвечающего уравнению (10), задаются как [4]-[6]

$Y_{1}={ }_{2} F_{1}\left(1+A, 1+B ; 2 ; 1-e^{-r / a}\right), \quad Y_{2}=\left.\left(1-e^{-r / a}\right)^{-1}{ }_{2} F_{1}\left(A, B ; \varepsilon ; 1-e^{-r / a}\right)\right|_{\varepsilon \rightarrow 0}$.

Хюльтеновская функция Грина уходящей волны имеет вид [3]

$$
G_{\mathrm{H}}^{(+)}\left(r, r^{\prime}\right)=-k^{-1} \Psi_{\mathrm{H}}^{(+)}\left(k, r_{<}\right) f_{\mathrm{H}}\left(k, r_{>}\right),
$$

где $r_{>}$и $r_{<}-$минимальное и максимальное значения $r$,

$$
\Psi_{\mathrm{H}}^{(+)}(k, r)=\frac{\Gamma(A+1) \Gamma(B+1)}{\Gamma(C)} a k e^{i k r}\left(1-e^{-r / a}\right)_{2} F_{1}\left(A+1, B+1 ; 2 ; 1-e^{-r / a}\right)
$$


И

$$
f_{\mathrm{H}}(k, r)=e_{2}^{i k r} F_{1}\left(A, B ; C ; e^{-r / a}\right), \quad C=1-2 i a k .
$$

Здесь $\Psi_{\mathrm{H}}^{(+)}(k, r)$ и $f_{\mathrm{H}}(k, r)$ обозначают физическое и нерегулярное $\mathrm{S}$-волновые решения уравнения с потенциалом Хюльтена [7], [8].

Подставляя (14) в уравнение (8) и совершая преобразование Лапласа, получаем

$$
\begin{aligned}
\bar{F}(r, \beta)= & -\frac{e^{-i k r}}{a k}\left(1-e^{-r / a}\right)^{-1} \times \\
& \times\left[f_{\mathrm{H}}(k, r) \int_{0}^{r} d r^{\prime} e^{-\beta r^{\prime}} \Psi_{\mathrm{H}}^{(+)}\left(k, r^{\prime}\right)+\Psi_{\mathrm{H}}^{(+)}(k, r) \int_{r}^{\infty} d r^{\prime} e^{-\beta r^{\prime}} f_{\mathrm{H}}\left(k, r^{\prime}\right)\right] .
\end{aligned}
$$

Сравнивая выражения (12) и (17) для $\bar{F}(r, \beta)$ при $r=0$ и $r \rightarrow \infty$, получаем, что

$$
R_{1}=-\frac{\Gamma(A+1) \Gamma(B+1)}{\Gamma(C)(\beta-i k)}{ }_{3} F_{2}(A, B,(\beta-i k) a ;(\beta-i k) a+1, C ; 1), \quad R_{2}=0 .
$$

Из соотношений (8), (12) и (18) имеем

$$
\begin{aligned}
& G_{\mathrm{H}}^{(+)}(r, \beta)=a e^{i k r}\left(1-e^{-r / a}\right) \times \\
& \times\left[-\frac{\Gamma(A+1) \Gamma(B+1)}{\Gamma(C)(\beta-i k)} \times\right. \\
& \quad \times{ }_{3} F_{2}(A, B,(\beta-i k) a ;(\beta-i k) a+1, C ; 1){ }_{2} F_{1}\left(A+1, B+1 ; 2 ; 1-e^{-r / a}\right)+ \\
& \left.\quad+a \sum_{n=0}^{\infty} \frac{\Gamma(n+1-(\beta+i k) a)}{\Gamma(1-(\beta+i k) a) n !} f_{n+1}\left(A+1, B+1 ; 2 ; 1-e^{-r / a}\right)\right] .
\end{aligned}
$$

В приложении мы показываем, что можно получить выражение (19) из соотношений (4), (14) и (15), (16) прямым интегрированием.

Двукратное преобразование Лапласа можно легко вывести, проинтегрировав выражение (19) с заменой независимой переменной $z=1-e^{-r / a}$. Это дает

$$
\begin{aligned}
& G_{\mathrm{H}}^{(+)}(\alpha, \beta)=a^{3} \sum_{n=0}^{\infty} \frac{\Gamma(n+1-(\beta+i k) a) \Gamma((\alpha-i k) a)}{\Gamma(1-(\beta+i k) a) \Gamma(n+3+(\alpha-i k) a)} \times \\
& \times{ }_{3} F_{2}(1, n+A+2, n+B+2 ; n+2, n+3+(\alpha-i k) a ; 1)- \\
&-a^{2} \frac{\Gamma(A+1) \Gamma(B+1) \Gamma((\alpha-i k) a) \Gamma((\alpha+i k) a)}{(\beta-i k) \Gamma(C) \Gamma(1+(\alpha-i k) a-A) \Gamma(1+(\alpha-i k) a-B)} \times \\
& \quad \times{ }_{3} F_{2}(A, B,(\beta-i k) a ;(\beta-i k) a+1, C ; 1) .
\end{aligned}
$$

Используя формулу [5], [6], [9]

$$
{ }_{3} F_{2}(a, b, c ; e, f ; 1)=\frac{\Gamma(s) \Gamma(f) \Gamma(e)}{\Gamma(a) \Gamma(s+c) \Gamma(s+b)}{ }_{3} F_{2}(s, e-a, f-a ; s+b, s+c ; 1),
$$

где $s=e+f-a-b-c$, и равенство

$$
{ }_{2} F_{1}(a, b, c ; 1)=\frac{\Gamma(c) \Gamma(c-a-b)}{\Gamma(c-a) \Gamma(c-b)},
$$


мы приходим к следующему выражению:

$$
\begin{aligned}
G_{\mathrm{H}}^{(+)}(\alpha, \beta)= & -a^{3} \frac{\Gamma(1+B) \Gamma((\alpha-i k) a)}{(1+A) \Gamma(1-(\beta+i k) a) \Gamma(2+(\alpha-i k) a)} \times \\
& \times \sum_{n=0}^{\infty} \frac{\Gamma(n+2) \Gamma(n+1-(\beta+i k) a)}{\Gamma(n+1) \Gamma(n+B+2)} \times \\
& \quad \times{ }_{3} F_{2}(-n, 1+A, 1-(\alpha-i k) a-B ; A+2,2+(\alpha-i k) a ; 1) .
\end{aligned}
$$

После суммирования ряда и перегруппировки членов имеем

$$
\begin{aligned}
G_{\mathrm{H}}^{(+)}(\alpha, \beta)= & \frac{a^{3} B \Gamma((\alpha-i k) a) \Gamma(B+(\beta+i k) a-1)}{(1+A) \Gamma(2+(\alpha-i k) a) \Gamma(B+(\beta+i k) a+1)} \times \\
& \times{ }_{4} F_{3}(2,1+A, 1-(\beta+i k) a, 1+(\alpha-i k) a-B ; \\
& A+2,2+(\alpha-i k) a, 2-B-(\beta+i k) a ; 1) .
\end{aligned}
$$

Здесь ${ }_{4} F_{3}(\cdot)$ - вполне уравновешенная гипергеометрическая функция.

Обозначим через $D^{(+)}(k)=1-\lambda G_{\mathrm{H}}^{(+)}(\alpha, \beta)$ определитель Фредгольма, ассоциированный с физическим решением $\Psi_{\mathrm{H}}^{(+)}(k, r)$. В случае локального потенциала определитель Фредгольма $D^{(+)}(k)$ совпадает с функцией Йоста $f(k)$. В случае нелокального потенциала или комбинации локального и нелокального потенциалов функции $D^{(+)}(k)$ и $f(k)$ не равны друг друг другу, а связаны соотношением $f(k)=D^{(+)}(k) / D(k)[10]-[12]$. Здесь $D(k)$ - определитель Фредгольма, ассоциированный с регулярным или нерегулярным решением, который всегда является действительным числом. В общем случае функция Йоста $f(k)$ и определитель Фредгольма $D^{(+)}(k)$ являются комплексными величинами. При этом фаза величины $f(k)$ совпадает с фазой величины $D^{(+)}(k)$. Более того, фаза функции Йоста равна сдвигу $\delta(k)$ фазы рассеяния, взятому со знаком минус. Таким образом, формула (22) дает удобный способ вычисления сдвига фазы рассеяния.

\section{3. КУЛОНОВСКИЙ ПРЕДЕЛ И СДВИГ ФАЗЫ РАССЕЯНИЯ}

Вычислим для проверки кулоновский предел полученных нами выражений.

Пусть $a \rightarrow \infty$ и $V_{0} \rightarrow 0$ так, что их произведение остается постоянным, $a V_{0}=2 k \eta$, где $\eta$ - параметр Зоммерфельда. В пределе $a \rightarrow \infty$ имеем $A \sim i \eta, B \sim i \eta-2 i a k$ и $C \sim 2 i a k$. Используя преобразование Томае функции ${ }_{3} F_{2}(\cdot)$ [4]-[6], [9],

$$
{ }_{3} F_{2}(a, b, c ; e, f ; 1)=\frac{\Gamma(e) \Gamma(s)}{\Gamma(e-a) \Gamma(s+a)}{ }_{3} F_{2}(a, f-b, f-c ; f, s+a ; 1),
$$

где $s=e+f-a-b-c$, и соотношения

$$
\begin{gathered}
\lim _{z \rightarrow \infty} \frac{\Gamma(z+\alpha)}{\Gamma(z+\beta)}=z^{\alpha-\beta}\left[1+O\left(z^{-1}\right)\right], \quad|\arg (z)|<\pi, \\
\lim _{\alpha \rightarrow \infty}\left\{\alpha^{\sigma} f_{\sigma}(\alpha, \beta ; \gamma ; z / \alpha)\right\}=\theta_{\sigma}(\beta, \gamma ; z),
\end{gathered}
$$

выводим искомое выражение для функции $G_{\mathrm{C}}^{(+)}(r, \beta)$, представляющей собой результат однократного преобразования кулоновской функции Грина, умноженной 
на потенциал Ямагучи [11], [12]. В кулоновском пределе функция (22) принимает вид [13]-[15]

$$
\begin{aligned}
\left.G_{\mathrm{H}}^{(+)}(\alpha, \beta)\right|_{a \rightarrow \infty} & =G_{\mathrm{C}}^{(+)}(\alpha, \beta)= \\
& =-\frac{(\alpha+\beta)^{-1}}{(1+i \eta)(\alpha-i k)(\beta-i k)}{ }_{2} F_{1}\left(1, i \eta ; 2+i \eta ; \frac{(\alpha+i k)(\beta+i k)}{(\alpha-i k)(\beta-i k)}\right) .
\end{aligned}
$$

В пределе $V_{0} \rightarrow 0$ формулы (19) и (22) воспроизводят результаты для случая чистого потенциала Ямагучи [7], [16], [17].

Опираясь на выражение $(22)$ и определение детерминанта Фредгольма $D^{(+)}(k)$ мы рассчитали величину сдвига фазы рассеяния $\delta_{\mathrm{HY}}(k)$ в системах $\alpha-p, \alpha-\mathrm{He}^{3}$ и $\alpha-\mathrm{H}^{3}$, а также величину $\delta_{Y}(k)$ для системы $\alpha-n$. При расчете мы использовали такие значения параметров: $\hbar^{2} / 2 m=25.92 \mathrm{MэВ} \cdot \Phi_{\mathrm{M}^{2}}^{2}$ для системы $\alpha$-нуклон, $\hbar^{2} / 2 m=24.190833 \mathrm{MэB} \cdot \Phi_{\mathrm{M}^{2}}$ для системы $\alpha$-ядро и $V_{0} a=0.03472 \Phi_{\mathrm{M}}{ }^{-1}$ для системы $\alpha$-протон, $V_{0} a=0.1182 \Phi_{\mathrm{M}}^{-1}$ для системы $\alpha$-гелий, $V_{0} a=0.0591 \Phi_{\mathrm{M}}^{-1}$ для системы $\alpha$-водород [18], [19]. Другие параметры представлены в табл. 1.

ТАБЛицА 1

\begin{tabular}{|c|c|c|}
\hline Система & $\lambda\left(\Phi_{\mathrm{M}}{ }^{-3}\right)$ & $\alpha=\beta\left(\Phi_{\mathrm{M}}{ }^{-1}\right)$ \\
\hline$\alpha-n$ & -9.995 & 1.2 \\
\hline$\alpha-p$ & -13.56 & 1.3 \\
\hline$\alpha-\mathrm{H}^{3}$ & -12.5 & 0.75 \\
\hline$\alpha-\mathrm{He}^{3}$ & -4.7 & 0.693 \\
\hline
\end{tabular}

Для данных значений параметров мы провели расчет величины сдвига фазы рассеяния в диапазоне лабораторных энергий $E_{\mathrm{Lab}}$ между 0 и $12 \mathrm{MэB.} \mathrm{Результаты} \mathrm{рас-}$ чета отражены на рис. 1 и 2. При указанных значениях параметров взаимодействия была рассчитана парциальная волновая функция с $\ell=0$ и проведено ее сравнение со стандартными результатами [20]-[27]. Следует заметить, что фазовый сдвиг, вычисленный нами для рассматриваемых систем, хорошо согласуется с результатами работ [20]-[24].

Недавно в работе [20] было получено достаточно обоснованное описание упругого рассеяния тритона, опирающееся на использовании глобального потенциала оптической модели. В работе [22] с использованием потенциала оптической модели и в работе [21] в рамках формализма унитарного корреляционного оператора изучались процессы упругого рассеяния при энергиях ниже 20 МэВ в системах $\alpha-n$ и $\alpha-p$; было получено хорошее согласие с экспериментальными данными [25]. В недавнем прошлом в работе [20] были рассчитаны фазы упругого рассеяния в системах $\alpha-\mathrm{He}^{3}$ при низких энергиях, при этом использовалась комбинация потенциала простого взаимодействия двух тел и потенциала двойной свертки; было достигнуто хорошее согласие с экспериментальными данными [23], [26], [27]. 


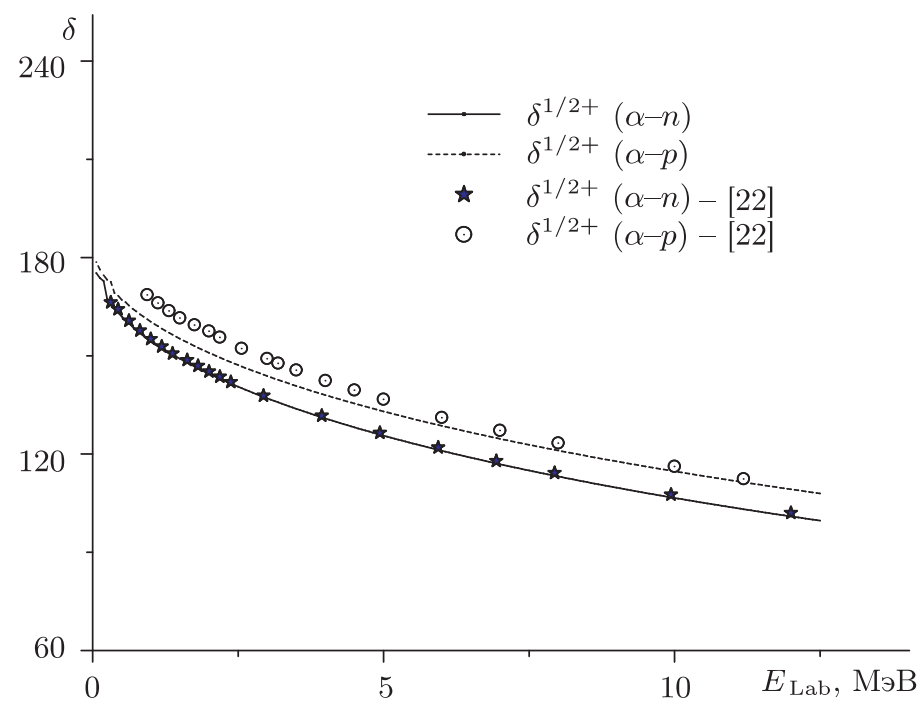

Рис. 1. Фазовый сдвиг $\delta$ (в градусах) как функция от лабораторной энергии для процесса рассеяния в системе $\alpha$-нуклон.

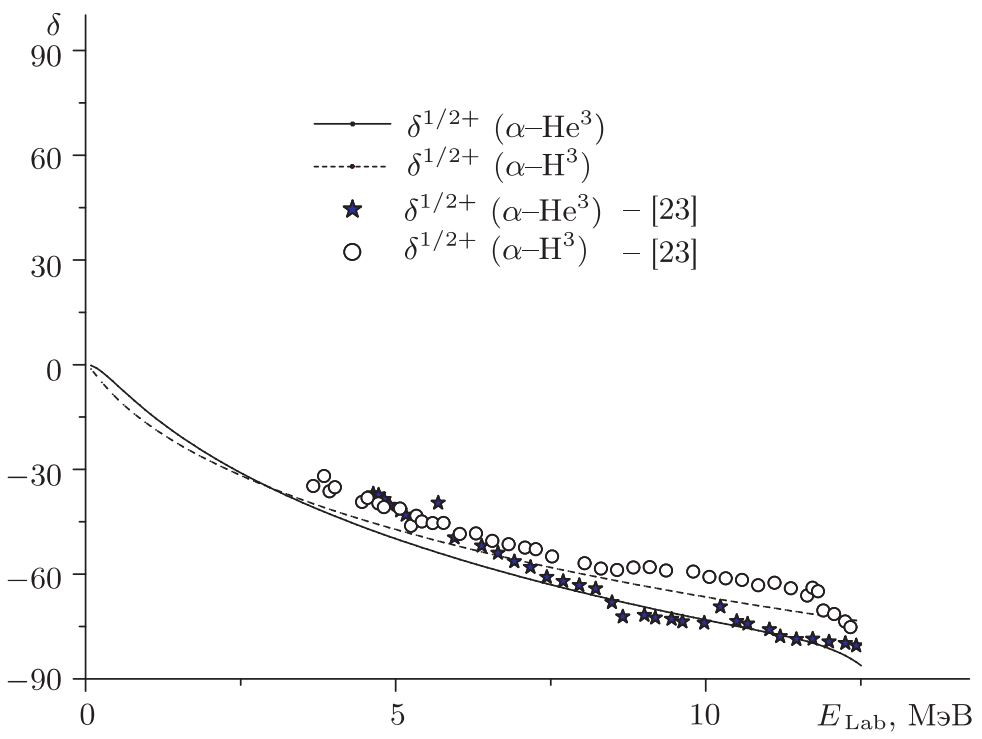

Рис. 2. Фазовый сдвиг $\delta$ (в градусах) как функция от лабораторной энергии для процесса рассеяния в системах $\alpha-\mathrm{He}^{3}$ и $\alpha-\mathrm{H}^{3}$.

Полученные нами значения фазового сдвига $\delta^{1 / 2+}$ для систем $\alpha-\mathrm{He}^{3}$ и $\alpha-\mathrm{H}^{3}$ слегка отличаются от результатов работы [23] в диапазоне энергий ниже 5 МэВ и хорошо согласуются при энергиях от 5 МэВ до 12 МэВ. С другой стороны, значения фазового сдвига $\delta^{1 / 2+}$ для системы $\alpha-\mathrm{He}^{3}$ хорошо выдерживают сравнение с результатом работы [20]. 


\section{4. ЗАКЛЮЧЕНИЕ}

Замкнутые выражения для преобразования Лапласа кулоновской функции Грина в задачах рассеяния на сумме кулоновского и нелокального сепарабельного потенциалов ранее были опубликованы в ряде работ (см., например, [11], [12], [28], [29]). В нашей статье мы представили результаты аналогичного исследования, проведенного с использованием потенциала Хюльтена вместо потенциала чисто кулоновского взаимодействия. Мы записали двукратное преобразование Лапласа для хюльтеновской функции Грина в его наиболее сжатом виде, куда входит только функция ${ }_{4} F_{3}(\cdot)$, в то время как аналогичные формулы из работы [7] содержат как функцию ${ }_{5} F_{4}(\cdot)$, так и две функции ${ }_{4} F_{3}(\cdot)$. K сожалению, нам не удалось сопоставить наше выражение для $G_{\mathrm{H}}^{(+)}(\alpha, \beta)$ с формулой из работы [7].

Один из авторов настоящей статьи в работе [30] рассматривал сумму потенциала Хюльтена и сепарабельного потенциала, чтобы получить замкнутое выражение для S-волнового фазового сдвига в рамках метода фазовых функций, примененного к модифицированным по Хюльтену сепарабельным взаимодействиям. В результате было получено хорошее согласие с данными о фазовом сдвиге при рассеянии нуклон-нуклон.

Было бы желательно распространить наши результаты на высшие парциальные волны. Такие попытки требуют получения аналитических решений для потенциала Хюльтена при $\ell>0$. Решения для высших парциальных волн в задаче с потенциалом Хюльтена можно построить в рамках формализма суперсимметрии, инспирированного методом факторизации [8], [18], [31]. Мы активно работаем над обобщением на случай высших парциальных волн преобразований функции Грина в задаче с суммой потенциала Хюльтена и сепарабельного нелокального потенциала и представим результаты в нашей будущей публикации.

Потенциал Хюльтена часто используется как модель экранированного кулоновского взаимодействия с обрезанием [32], [33]. Поскольку влияние экранирования неизбежно сказывается на теоретических результатах и интерпретации экспериментальных данных по рассеянию заряженных адронов, мы ожидаем, что представленный в настоящей статье анализ будет интересен широкому кругу физиков. Наши результаты легко можно распространить на более сложные потенциалы, и ограничение случаем симметричных потенциалов не является таким уж непреодолимым. Важно располагать альтернативными подходами к проблеме, поэтому мы надеемся, что представленный метод заслуживает внимания.

ПРИЛОЖЕНИЕ 1

\section{Вывод уравнения (19) путем прямого интегрирования}

Функция Грина (14) может быть записана через регулярную функцию Грина следующим образом:

$$
\begin{aligned}
G_{\mathrm{H}}^{(+)}\left(r, r^{\prime}\right)= & G_{\mathrm{H}}^{(\mathrm{R})}\left(r, r^{\prime}\right)-\frac{\Gamma(A+1) \Gamma(B+1)}{\Gamma(C)} a e^{i k\left(r+r^{\prime}\right)}\left(1-e^{-r / a}\right) \times \\
& \times{ }_{2} F_{1}\left(A+1, B+1 ; 2 ; 1-e^{-r / a}\right){ }_{2} F_{1}\left(A, B ; C ; e^{-r^{\prime} / a}\right),
\end{aligned}
$$


где регулярная хюльтеновская функция Грина имеет вид

$$
\begin{aligned}
G_{\mathrm{H}}^{(\mathrm{R})}\left(r, r^{\prime}\right)= & \frac{\Gamma(A+1) \Gamma(B+1)}{\Gamma(C)} a e^{i k\left(r+r^{\prime}\right)}\left[\left(1-e^{-r / a}\right) \times\right. \\
& \quad \times{ }_{2} F_{1}\left(A+1, B+1 ; 2 ; 1-e^{-r / a}\right){ }_{2} F_{1}\left(A, B ; C ; e^{-r^{\prime} / a}\right)- \\
& \left.-\left(1-e^{-r^{\prime} / a}\right){ }_{2} F_{1}\left(A+1, B+1 ; 2 ; 1-e^{-r^{\prime} / a}\right){ }_{2} F_{1}\left(A, B ; C ; e^{-r / a}\right)\right] .
\end{aligned}
$$

Преобразуя ${ }_{2} F_{1}\left(A, B ; C ; e^{-r / a}\right)$ с использованием рекуррентного соотношения [5], [6]

$$
\begin{aligned}
{ }_{2} F_{1}(a, b ; c ; z)= & \frac{\Gamma(c) \Gamma(c-a-b)}{\Gamma(c-a) \Gamma(c-b)}{ }_{2} F_{1}(a, b ; a+b-c+1 ; 1-z)+ \\
& +(1-z)^{c-a-b} \frac{\Gamma(c) \Gamma(a+b-c)}{\Gamma(a) \Gamma(b)}{ }_{2} F_{1}(c-a, c-b ; c-a-b+1 ; 1-z),
\end{aligned}
$$

получаем

$$
\begin{aligned}
G_{\mathrm{H}}^{(\mathrm{R})}\left(r, r^{\prime}\right)= & a e^{i k\left(r+r^{\prime}\right)} \times \\
& \times \lim _{\varepsilon \rightarrow 0}\left[\left(1-e^{-r / a}\right){ }_{2} F_{1}\left(A+1, B+1 ; 2 ; 1-e^{-r / a}\right){ }_{2} F_{1}\left(A, B ; \varepsilon ; 1-e^{-r^{\prime} / a}\right)-\right. \\
& \left.\quad-\left(1-e^{-r^{\prime} / a}\right){ }_{2} F_{1}\left(A+1, B+1 ; 2 ; 1-e^{-r^{\prime} / a}\right){ }_{2} F_{1}\left(A, B ; \varepsilon ; 1-e^{-r / a}\right)\right] .
\end{aligned}
$$

Комбинируя соотношения (5), (П.1) и (П.2), приходим к выражению

$$
G_{\mathrm{H}}^{(+)}(\beta, r)=I_{1}(\beta, r)-I_{2}(\beta, r),
$$

где

$$
\begin{aligned}
I_{1}(\beta, r)= & \int_{0}^{r} d r^{\prime} G_{\mathrm{H}}^{(\mathrm{R})}\left(r, r^{\prime}\right) e^{-\beta r} \\
I_{2}(\beta, r)= & a e^{i k r}\left(1-e^{-r / a}\right) \frac{\Gamma(A+1) \Gamma(B+1)}{\Gamma(C)}{ }_{2} F_{1}\left(A+1, B+1 ; 2 ; 1-e^{-r / a}\right) \times \\
& \times \int_{0}^{\infty} d r^{\prime} e^{-(\beta-i k) r^{\prime}}{ }_{2} F_{1}\left(A, B ; C ; e^{-r^{\prime} / a}\right) .
\end{aligned}
$$

Используем замену переменной $z=e^{-r^{\prime} / a}$ и найдем интеграл в (П.5). В результате получим

$$
\begin{aligned}
I_{2}(\beta, r)= & a e^{i k r}\left(1-e^{-r / a}\right) \frac{\Gamma(A+1) \Gamma(B+1)}{(\beta-i k) \Gamma(C)} \times \\
& \times{ }_{2} F_{1}\left(A+1, B+1 ; 2 ; 1-e^{-r / a}\right){ }_{3} F_{2}(A, B,(\beta-i k) a ; c ;(\beta-i k) a+1 ; 1) .
\end{aligned}
$$

Подставим выражение (П.2) в (П.4), сделаем замену переменной $z^{\prime}=\left(1-e^{-r^{\prime} / a}\right)$ и используем разложение в ряд

$$
\left(1-z^{\prime}\right)^{(\beta+i k) a-1}=\sum_{n=0}^{\infty} \frac{\Gamma(n+1-(\beta+i k) a)}{\Gamma(1-(\beta+i k) a)} \frac{z^{\prime n}}{n !} .
$$


Формула (П.4) дает

$$
I_{1}(\beta, r)=a^{2} e^{i k r}\left(1-e^{-r / a}\right) \sum_{n=0}^{\infty} \frac{\Gamma(n+1-(\beta+i k) a)}{\Gamma(1-(\beta+i k) a) n !} f_{n+1}\left(A+1, B+1 ; 2 ; 1-e^{r / a}\right) .
$$

При выводе этого выражения мы использовали стандартный интеграл [4]

$$
\begin{aligned}
& f_{\sigma}(a, b ; c ; z)=\frac{1}{c-1} \times \\
& \times\left[{ }_{2} F_{1}(a, b ; c ; z) \int_{0}^{z} d z^{\prime} z^{\sigma-1}\left(1-z^{\prime}\right)^{a+b-c}{ }_{2} F_{1}\left(a-c+1, b-c+1 ; 2-c ; z^{\prime}\right)-\right. \\
& \left.\quad-z^{1-c}{ }_{2} F_{1}(a-c+1, b-c+1 ; 2-c ; z) \int_{0}^{z} d z^{\prime} z^{\prime \sigma+c-2}\left(1-z^{\prime}\right)^{a+b-c}{ }_{2} F_{1}\left(a, b ; c ; z^{\prime}\right)\right] .
\end{aligned}
$$

Комбинация соотношений (П.6) и (П.8) в комбинации с равенством (П.3) дает искомое выражение для $G_{\mathrm{H}}^{(+)}(\beta, r)$.

\section{Список литературы}

[1] L. Hulthén, "Über die Eigenlösungen der Schrödinger-Gleichung des Deuterons", Ark. Mat. Astron. Fysik A, 28:5 (1942), 1-12.

[2] Y. Yamaguchi, "Two-nucleon problem when the potential is nonlocal but separable. I", Phys. Rev., 95:6 (1954), 1628-1634.

[3] Р. Ньютон, Теория рассеяния волн и частии, Мир, М., 1969.

[4] A. W. Babister, Transcendental Functions Satisfying Non-Homogeneous Linear Differential Equations, MacMillan, New York, 1967.

[5] L. J. Slater, Generalized Hypergeometric Functions, Cambridge Univ. Press, London, 1966.

[6] Г. Бейтмен, А. Эрдейи, Высшие трансцендентные функции, т. 1, Мир, Наука, 1973.

[7] H. van Haeringen, Charged Particle Interactions. Theory and Formulas, The Coulomb Press, Leyden, 1985.

[8] U. Laha, C. Bhattacharyya, K. Roy, B. Talukdar, "Hamiltonian hierarchy and the Hulthén potential", Phys. Rev. C, 38:1 (1988), 558-560.

[9] W. N. Balley, Generalised Hypergeometric Series, Cambridge Univ. Press, London, 1935.

[10] C. S. Warke, R. K. Bhaduri, "On the Jost function of a non-local potential", Nucl. Phys. A, 162:2 (1971), 289-294.

[11] B. Talukdar, U. Laha, T. Sasakawa, "Green's function for motion in Coulomb-modified separable nonlocal potentials", J. Math. Phys, 27:10 (1986), 2080-2086.

[12] U. Laha, B. J. Roy, B. Talukdar, "Transforms of the Coulomb Green function by the form factor of the Graz potential", J. Phys. A: Math. Gen., 22:17 (1989), 3597-3604.

[13] B. Talukdar, U. Laha, S. R. Bhattaru, "Double Laplace transform of the Coulomb Green function", J. Phys. A: Math. Gen., 18:7 (1985), L359-362.

[14] U. Laha, J. Bhoi, "Off-shell Jost solutions for Coulomb and Coulomb-like interactions in all partial waves", J. Math. Phys., 54:1 (2013), 013514, 22 pp.

[15] U. Laha, J. Bhoi, "Integral transform of the Coulomb Green's function by the Hankel function and off-shell scattering", Phys. Rev. C, 88:6 (2013), 064001, 10 pp.

[16] D. K. Ghosh, S. Saha, K. Niyogi, B. Talukdar, "Laplace transform method for off-shell scattering on nonlocal potentials", Czech. J. Phys. B, 33:5 (1983), 528-539.

[17] J. Bhoi, U. Laha, "Integral transforms and their applications to scattering theory", Int. J. Appl. Phys. Math., 4:6 (2014), 386-405. 
[18] U. Laha, J. Bhoi, "Higher partial-wave potentials from supersymmetry-inspired factorization and nucleon-nucleus elastic scattering", Phys. Rev. C, 91:3 (2015), 034614, 6 pp.

[19] J. Bhoi, U. Laha, "Elastic scattering of light nuclei through a simple potential model", Phys. Atomic Nucl., 79:4 (2016), 370-374.

[20] P. Mohr, "Low-energy ${ }^{3} \mathrm{He}(\alpha, \alpha){ }^{3} \mathrm{He}$ elastic scattering and the ${ }^{3} \mathrm{He}(\alpha, \gamma){ }^{7} \mathrm{Be}$ reaction", Phys. Rev. C, 79:6 (2009), 065804, 10 pp.

[21] J. Dohet-Eraly, D. Baye, "Microscopic cluster model of $\alpha+n, \alpha+p, \alpha+{ }^{3} \mathrm{He}$, and $\alpha+\alpha$ elastic scattering from a realistic effective nuclear interaction", Phys. Rev. C, 84:1 (2011), 014604, 13 pp.

[22] G. R. Satchler, L. W. Owen, A. J. Elwin, G. L. Morgan, R. L. Walter, "Microscopic study of nucleon $-{ }^{4}$ He scattering and effective nuclear potentials", Nucl. Phys. A, 112:1 (1968), $1-31$.

[23] R. J. Spiger, T. A. Tombrello, "Scattering of $\mathrm{He}^{3}$ by $\mathrm{He}^{4}$ and of $\mathrm{He}^{4}$ by Tritium", Phys. Rev., 163:4 (1967), 964-984.

[24] Y. Li Xu, H. Rui Guo, Y. Lu Han, Q. Biao Shen, "Applicability of the systematic helium-3 potential for triton-nucleus reactions", Internat. J. Modern Phys. E, 24:1 (2015), 1550005, $14 \mathrm{pp}$.

[25] G. L. Morgan, R. L. Walter, "Neutron-Helium Interaction. II. Angular Distributions and phase shifts from 0.2 to $7.0 \mathrm{MeV}$ ", Phys. Rev., 168:4 (1968), 1114-1129.

[26] W. R. Boykin, S. D. Baker, D. M. Hardy, "Scattering of ${ }^{3} \mathrm{He}$ and ${ }^{4} \mathrm{He}$ from polarized ${ }^{3} \mathrm{He}$ between 4 and $10 \mathrm{MeV}$ ", Nucl. Phys. A, 195:1 (1972), 241-249.

[27] D. M. Hardy, R. J. Spiger, S. D. Baker, Y. S. Chen, T. A. Tombrello, "Scattering of ${ }^{3}$ He and ${ }^{4} \mathrm{He}$ from polarized ${ }^{3} \mathrm{He}$ between 7 and $18 \mathrm{MeV}$ ", Nucl. Phys. A, 195:1 (1972), 250-256.

[28] H. van Haeringen, "Off-shell $T$ matrix for Coulomb plus simple separable potentials for all $l$ in closed form", J. Math. Phys., 24:5 (1983), 1274-1281.

[29] B. Talukdar, D. K. Ghosh, T. Sasakawa, "Coulomb-modified nuclear scattering. III", J. Math. Phys, 25:2 (1984), 323-326.

[30] U. Laha, A. K. Jana, T. K. Nandi, "Phase-function method for Hulthén-modified separable potentials", Pramana, 37:5 (1991), 387-393.

[31] C. V. Sukumar, "Supersymmetry, factorisation of the Schrodinger equation and a Hamiltonian hierarchy", J. Phys. A: Math. Gen., 18:2 (1985), L57-L62.

[32] J. Lindhard, A. Winther, "Transient fields acting on heavy ions during slowing-down in magnetized materials", Nucl. Phys. A, 166:3 (1971), 413-435.

[33] W. D. Kraeft, M. Luft, A. A. Mihajlov, "Scattering properties and electrical conductivity for the Coulomb cut-off potential", Physica A: Stat. Mech. Appl., 120:1-2 (1983), 263-278.

Поступила в редакцию 18.10.2016, после доработки 24.11.2016 